\title{
Übersetzen im Fremdsprachenunterricht
}

\author{
Claudia Ascher \\ Universidade de Coimbra, Portugal \\ ascherclaudia@gmail.com
}

\begin{abstract}
Übersetzen als Aktivität im Fremdsprachenunterricht (FSU) wurde und wird immer wieder kontrovers diskutiert. Besonders als Bestandteil der sogenannten GrammatikÜbersetzungs-Methode (Richards \& Rodgers, 2001, pp. 5-7) wurde daran unter anderem kritisiert, dass es sich um eine rein kognitive Auseinandersetzung mit der Sprache handelt, die deshalb keinen kommunikativen und emotionalen Zugang ermöglicht.
\end{abstract}

Seit der zweiten Hälfte des letzten Jahrhunderts und zunehmend in den letzten Jahren scheint das Übersetzen im FSU und die Verwendung der Lernersprache (LS) jedoch eine Renaissance zu erleben (vgl. beispielsweise Butzkamm \& Caldwell, 2009, Cook, 2010, Hall \& Cook, 2012). An der Universität Coimbra (Portugal) ist es seit vielen Jahren fester Bestandteil des Deutschunterrichts im Abschlussjahr des Studiengangs Línguas Modernas, und die Erfahrung zeigt, dass das Übersetzen im FSU nicht nur die Kompetenz in der Fremdsprache (und auch in der Muttersprache) fördert, sondern ebenfalls dazu geeignet ist, die Lerner aktiv in den Lernprozess einzubinden und dabei auch auf einer emotionaler Ebene anzusprechen.

Nach einer historisch-theoretischen Einbettung des Themas wird im vorliegenden Beitrag ein Beispiel für einen erfolgreichen und stimulierenden Einsatz von Übersetzungsaktivitäten im FSU vorgestellt.

Schlüsselwörter: Übersetzen, Fremdsprachenunterricht, Deutsch als Fremdsprache

\section{Abstract}

Translation as an activity in foreign language teaching has been a controversial topic of discussions. Especially when applied using the Grammar-Translation-Method (Richards \& Rodgers, 2001, pp. 5-7) it has been criticized, among other things, for being an exclusively cognitive method of language learning which does not incorporate a communicative or an emotional approach.

Since the second half of the last century and increasingly in the last few years, translation in foreign language teaching and the use of the learner's mother-tongue 
ASCHER, Claudia - Übersetzen im Fremdsprachenunterricht. Para lá da tarefa: implicar os estudantes na aprendizagem de línguas estrangeiras no ensino superior. Porto: FLUP, 2019, pp. 25-43 DOI: https://doi.org/10.21747/9789898969217/paraa2

seems to be experiencing a renaissance (see i.e. Butzkamm \& Caldwell 2009, Cook 2010, Hall \& Cook 2012). At the University of Coimbra (Portugal), translation has been a core part of German as a Foreign Language classes for final year students of the Bachelor's course in Línguas Modernas for many years. Our experience shows that translation in foreign language teaching is not only a tool to improve the learner's competence in the foreign language (and, also, in their mother-tongue), but also a good way to actively include the learner in the learning process and to involve him or her emotionally as well.

After giving a historical-theoretical framework of the topic, the present work will show an example of successful and stimulating use of translation-exercises in the foreign language classroom.

Keywords: Translation, Foreign language teaching, German as a foreign language

\section{1 - Einleitung}

Als ich den Unterricht im letzten Jahr des Studienganges Línguas Modernas von einer Kollegin übernehmen sollte, da diese bald darauf in Rente ging, beschloss ich, zunächst bei ihr zu hospitieren, um ihre Unterrichtsmethoden besser kennen zu lernen und eventuell übernehmen zu können. Ich wusste, dass im Abschlussjahr ohne Lehrbuch unterrichtet wird, dafür aber die Arbeit mit authentischen (Hör)Texten und selbsterstelltem Material im Vordergrund steht - auf diese Aufgabe wollte ich so gut wie möglich vorbereitet sein.

Die Kollegin - selbst eine erfahrene Übersetzerin - erläuterte mir im Zuge dessen auch, dass sie von den drei Wochenstunden, die sie mit den Studenten zur Verfügung hatte, eine Stunde als Übersetzungseinheit reserviert hatte. Ich war diesem Vorhaben gegenüber skeptisch.

In meinem Masterstudium „Deutsch als Fremdsprache“ hatte ich verschiedenste Methoden des Fremdsprachenunterrichts (FSU) und deren Vor-und Nachteile kennen gelernt. Wenn die Aktivität „Übersetzen“ darin vorkam, dann in der Regel in einem negativen Kontext. Überhaupt wurde jeglicher Gebrauch der Lernersprache (LS) zumeist harsch zurückgewiesen. Wir wurden angehalten, den Unterricht möglichst vollständig in deutscher Sprache zu gestalten. An diesen Grundsatz hatte ich mich seit jeher gehalten und hatte auch Erfolg damit. Zwar kostete es viel Zeit und Geduld, aber auch der Nullanfängerunterricht, für den ich in Coimbra zuständig war (und teilweise immer noch bin), wurde und wird in deutscher Sprache unterrichtet. Das Ergebnis ist zumeist ein 
ASCHER, Claudia - Übersetzen im Fremdsprachenunterricht. Para lá da tarefa: implicar os estudantes na aprendizagem de línguas estrangeiras no ensino superior. Porto: FLUP, 2019, pp. 25-43 DOI: https://doi.org/10.21747/9789898969217/paraa2

solides Vertrauen der Studierenden in ihre eigenen Fähigkeiten beim Hörverstehen und beim Sprechen und eine positive Entwicklung dieser Fertigkeiten.

Die vorliegende Arbeit soll deshalb auch kein grundsätzlicher Angriff auf den einsprachig-kommunikativen Unterricht oder einen Gegenentwurf dazu sein, sondern eine Anregung darstellen, diese Lehrform mit lernersprachlichen Elementen zu erweitern und zu ergänzen. Im Sinne von Butzkamm \& Caldwell (2009, p. 13) suche ich damit eine Form der „new balance“ zwischen LS und Fremdsprache (FS), bei der die FS durchaus noch die vorherrschende Rolle im FSU spielt, wo aber auch die LS gezielte Verwendung findet. Eine Form davon können Übersetzungsübungen sein. Mithilfe eines konkreten Beispiels aus dem Unterricht möchte ich ein lehrpraktisches Verfahren für eine didaktisierte Form der Übersetzung vorstellen, die im besten Fall als Anregung für die Unterrichtsgestaltung von Kolleg*innen dienen kann. Zu Beginn werde ich zur Kontextualisierung einen kurzen historischen Überblick über Lehrmethoden im FSU geben und die Argumente umreißen, die für die Verwendung der LS und den Einsatz von Übersetzung im FSU sprechen.

\section{2 - Prämissen}

Dass Übersetzung Gegenstand der Fremdsprachdidaktik ist, kann seit der zweiten Hälfte des letzten Jahrhunderts nicht mehr ernsthaft in Frage gestellt werden, es gilt jedoch, verschiedene Funktionen, die sie in FSU haben kann zu unterscheiden:

Hinsichtlich der Funktion der Übersetzung ist es inzwischen üblich geworden, zwischen zwei Verwendungsweisen zu unterscheiden: Einerseits wird die Übersetzung als ein methodisches Mittel zur Festigung, Erweiterung und Prüfung sprachlicher Fertigkeiten angewendet, andererseits ist sie als eine eigene Fertigkeit selbst ein Übungs- und Unterrichtsziel. (Gündoğdu \& Büyüknisan, 2005, p. 188)

Wenn ich im Folgenden vom Übersetzen oder von Übersetzung im FSU spreche, dann unterscheide ich dieses Mittel in der Unterrichtsgestaltung grundsätzlich vom regulären Übersetzungsunterricht in der Übersetzerausbildung. Anders als bei letzterem, steht bei der Übersetzung im FSU ein utilitaristischer Ansatz im Vordergrund: Die Lehrform wird hier als ein möglicher, alternativer Zugang zur FS ge- und benutzt. Andere übersetzungswissenschaftliche Kompetenzen, Übersetzungsstrategien und relevante Techniken, die im regulären Übersetzungsunterricht im Zentrum der Aufmerksamkeit stehen, werden hingegen weitestgehend vernachlässigt, denn das Ziel von 
ASCHER, Claudia - Übersetzen im Fremdsprachenunterricht. Para lá da tarefa: implicar os estudantes na aprendizagem de línguas estrangeiras no ensino superior.

Übersetzungsübungen im FSU ist nicht die grundständige Ausbildung von Übersetzern, sondern die Erweiterung der Kompetenzen in der FS. Trotz dieser notwendigen Differenzierung können Synergieeffekte genutzt werden: „Übersetzen als Übungsform und Übersetzen als Fertigkeit sind voneinander abgegrenzte Wirklichkeitsbereiche, können allerdings ,zusammenarbeiten' und voneinander profitieren“ (Rodrigues, 2000, p. 25). ${ }^{1}$ Auch die Förderung der Kompetenzen in der LS ist ein willkommener Nebeneffekt dieser Übungsform. Meine Perspektive ist dabei immer die Übersetzung von der LS in die FS, im konkreten Fall handelt es sich um die Übersetzung vom Portugiesischen ins Deutsche. Neben dieser konkreten Übungsform werde ich zudem einige Überlegungen zum Nutzen des allgemeinen Gebrauchs der LS im FSU einfließen lassen. Wie ich in der Einleitung bereits dargelegt habe, gilt die LS als Kommunikationsmittel im modernen FSU oft als nicht zulässig. Es gibt jedoch auch Stimmen, die einen (dosierten und gezielten) Gebrauch der LS als vorteilhaft bewerten. Da der Gebrauch der LS im FSU in unmittelbarem Zusammenhang mit der Benutzung der Übungsform "Übersetzung" steht, werde ich an passender Stelle ebenfalls auf diese Ansätze verweisen.

Die hier zu Grunde liegende Kombination von LS (Portugiesisch) und FS (Deutsch) ist auch unter dem Gesichtspunkt wichtig, dass Übersetzung im FS nicht für jedes Sprachenpaar in der gleicher Weise verwendet werden kann. Es ist einleuchtend, dass etwa ein japanischer Lerner, der Französisch lernen möchte vor anderen Herausforderungen steht als ein portugiesischer Lerner mit dem gleichen Ziel. Dementsprechend müssen auch die Lehr- und Unterrichtsmethoden angepasst werden. Der Kontext meiner Überlegungen ist eine weitestgehend homogene Lernergruppe: portugiesischsprachige Studenten, die Deutsch als eines der Kernfächer ihres Studiengangs Línguas Modernas gewählt haben und die sich bereits in einem fortgeschrittenen Studienjahr befinden, in der Regel im 3. Jahr ihrer licenciatura. Das

\footnotetext{
${ }^{1}$ House (2001) argumentiert in diesem Punkt anders und verlangt, dass „[w]enn Übersetzungen im Deutschunterricht [...] verwendet werden, so ist es unabdingbar, sie nicht als didaktisches Mittel für andere Zwecke, sondern genuin als Übersetzungen, d.h. als kommunikative Handlungen zu verwenden, die eine reale kommunikative Handlung erfüllen." (House, 2001, p. 265). Dieser Auffassung kann entgegengehalten werden, dass eine Sprachhandlung in einer Unterrichtssituation zwar authentische Situationen imitieren, selbst aber nie eine solche genuin sein kann. Trotzdem ist eine Übersetzung meiner Auffassung nach eine authentische sprachliche Handlung, auch wenn sie in didaktisierter Form aufbereitet wird. Eine utilitaristisch-didaktische Verwendung von Übersetzung im FSU und Authentizität schließen sich aber nicht aus, wie ich in Punkt 4.3 ausführen werde, da authentische Kommunikation im Lernkontext und nicht in der „Echtheit" der verwendeten Materialen oder Aufgabenstellungen begründet liegt, wie House sie fordert.
} 
ASCHER, Claudia - Übersetzen im Fremdsprachenunterricht.

Para lá da tarefa: implicar os estudantes na aprendizagem de línguas estrangeiras no ensino superior.

Porto: FLUP, 2019, pp. 25-43

DOI: https://doi.org/10.21747/9789898969217/paraa2

angestrebte Niveau in diesem Studienjahr ist der Abschluss der Stufe B.1 und der Beginn der Niveaustufe B.2. des gemeinsamen europäischen Referenzrahmens für Sprachen. In der Regel befindet sich die Mehrheit der Studierenden auf dem geforderten Niveau.

Eine „weitestgehend“ homogene Gruppe heißt im konkreten Fall, dass sich unter den Lernern auch Erasmus-Studenten, zumeist aus dem europäischen Ausland und auch wenige chinesische Studenten befanden. Aus diesem Grund spreche ich im vorliegenden Text auch nicht von L1 und L2, sondern von LS und FS. Die LS ist bei allen Teilnehmern Portugiesisch, wenn diese auch nicht zwingend die L1 sein muss und Deutsch ist die FS, die in der Regel eben nicht die L2 darstellt (diese Rolle übernimmt in der Mehrheit der Fälle das Englische), sondern die L3 oder L4. Mit der Verwendung der Termini LS und FS möchte ich dieser leichten Heterogenität in den Lernergruppen gerecht werden.

\section{3 - Methoden im FSU - ein historischer Abriss}

Der Hauptgrund, warum die Übersetzung als Übungsform im FSU so einen schlechten Ruf genießt, ist in der Regel die sogenannte ÜbersetzungsGrammatikmethode. Die in ihrer Anfangszeit auch als „preußische Methode“ bekannte Unterrichtsform (Richards \& Rogers, 2001, p. 5) war für das Erlernen der „toten“ Sprachen Latein und Altgriechisch konzipiert (vgl. House 2001, p. 258) und war dementsprechend auf einen rezeptiven Erwerb der FS ausgerichtet. ${ }^{2}$ Die Methode wurde später aber auch auf die Lehre lebender Sprachen übertragen. Ziel war es in erster Linie, Regelwissen über die grammatischen und syntaktischen Strukturen und lexikalisches Wissen der (schriftlichen) FS zu vermitteln, ohne dabei kommunikative Fertigkeiten oder mündlichen Sprachgebrauch zu lehren. Zur Erschließung und Analyse der grammatikalischen Strukturen und lexikalischen Äquivalente eignete sich neben der expliziten Grammatikvermittlung nach Vorstellung der Verfechter dieser Methode auch die Übersetzung in die (und auch aus der) LS von zumeist „isolierte[n], künstlich konstruierte[n] Sätze[n]“ (House, 2001, p. 258). Die Fertigkeiten „Sprechen“ und „Hören“ wurden nicht oder nur in geringem Umfang berücksichtigt, ein authentischer und handlungsorientierter Gebrauch der FS stand nicht im Vordergrund, spontane Äußerungen wurden nicht gefördert und auswendig Gelerntes nahm einen zentralen Platz in dieser Methode ein. Ziel war nicht der aktive und autonome Gebrauch einer FS,

\footnotetext{
2 Die Kritik, die an dieser Methode geübt wird, ist berechtigt, muss jedoch auch im Licht ihrer Zielsetzung und ihrer Ausrichtung auf nicht mehr gesprochene Sprachen bewertet werden.
} 
ASCHER, Claudia - Übersetzen im Fremdsprachenunterricht. Para lá da tarefa: implicar os estudantes na aprendizagem de línguas estrangeiras no ensino superior. Porto: FLUP, 2019, pp. 25-43 DOI: https://doi.org/10.21747/9789898969217/paraa2

sondern vielmehr ein kognitives und intellektuelles Training mit Hilfe eines logischsystematischen Zugangs zur Sprache, wobei Wert auf korrekte und normgerechte Anwendung des vermittelten Regelwissens und Eindeutigkeit in den lexikalischen Entsprechungen in LS und FS gelegt wurde. Kontextgebundenheit, Kreativität und ein emotional-spielerischer Ansatz des FS-Erwerbs gehörten nicht zu den Prämissen dieser Methode.

Es ist leicht ersichtlich, dass in einem modernen FSU, der lebende (und lebendige) Sprachen vermitteln will, Reformbedarf an dieser Methode bestand. Als Reaktion auf die Grammatik- Übersetzungsmethode entstanden zahlreiche behavioristische Lehrformen, die sich oft auf die „Direkte Methode“ stützten, welche in den Grundzügen von Maximilian Berlitz, dem Gründer der bis heute existierenden Sprachinstitute gleichen Namens (Richards \& Rodgers, 2001, pp. 11-14), konzipiert wurde. „Direkt" bezieht sich dabei auf zwei prinzipielle Merkmale dieser Methode: zum einen die Einsprachigkeit des Unterrichts, um einen unmittelbaren Zugang zur FS zu ermöglichen und zu fördern, ohne dabei mit der LS konfrontiert zu sein und zum anderen die kommunikative, handlungsorientierte Anwendung der FS. Die Übersetzung als methodische Form des Unterrichts wurde zurückgedrängt, da sie keine direkte Form des Sprachzugangs darstellt und auf Grund der innewohnenden Präferenz des Schriftlichen nicht in den Rahmen einer kommunikativen Ausrichtung der Kompetenzen passt, und verschwand fast vollständig aus dem FSU.

Ausgehend von diesem prinzipiellen Paradigmenwechsel wurden verschiedenste Lehrmethoden entwickelt. Es handelt sich dabei um teilweise sehr unterschiedliche Ansätze, denen aber allen gemeinsam ist, dass sie zum einen FS-zentriert sind, also die LS weitgehend aus dem Unterricht ausschließen und teilweise regelrechte „Verbote“ der LS im FS formulieren. Zum anderen verfolgen sie vor allen Dingen kommunikative Ziele, die häufig mit einem imitatorischen Zugang zur FS arbeiten. Eine analytisch-kognitive Durchdringung von Strukturen in der FS ist in der Regel nicht als zentraler Lehrinhalt vorgesehen.

Die unterschiedlichen Ansätze in der Fremdsprachendidaktik sind gut und ausführlich dokumentiert (bspw. Richards \& Rodgers, 2001, pp. 3-70, Haß 2010, Doff 2016), deshalb seien an dieser Stelle nur noch einmal stichpunktartig auf einige ausgewählte kommunikative Methoden verwiesen, bei den Bezeichnungen und inhaltlichen Beschreibungen orientiere ich mich dabei an der Ausführungen von Richards \& Rodgers (2001): 
ASCHER, Claudia - Übersetzen im Fremdsprachenunterricht.

Para lá da tarefa: implicar os estudantes na aprendizagem de línguas estrangeiras no ensino superior.

Porto: FLUP, 2019, pp. 25-43

DOI: https://doi.org/10.21747/9789898969217/paraa2

Oral Approach and Situational Language Teaching: Ausgehend von in der FS mündlich präsentierten Lerninhalten werden neue Inhalte situativ eingeführt und geübt. Erst danach erfolgt eine schriftliche Fixierung des dargebotenen Stoffes.

Audiolingual Approach: Der Ansatz konzentriert sich auf die Fertigkeiten „Sprechen“ und „Hören“, die LS darf im Unterricht nicht verwendet werden. Übersetzungsübungen sind in fortgeschrittenen Niveaus als literarische Übung zulässig.

Total Physical Response: Hier wird das Lernen durch motorische Aktivitäten unterstützt. Hören, das Zeigen von Gegenständen und Vorführen von Aktivitäten und die Wiederholung durch die Lernenden stehen im Mittelpunkt dieser Methode, die eine Imitation des muttersprachlichen Erwerbs sein soll. Die LS spielt keine Rolle.

Silent Way: Die Lehrkraft soll bei dieser Methode sehr zurückhaltend agieren, die Protagonisten sind die Lernenden. Auch hier spielen Mündlichkeit, Motorik und das Lernen von Vokabular anhand von gezeigten Objekten eine zentrale Rolle. LS und Übersetzung finden keine Berücksichtigung, auch deshalb, weil es in erster Linie um den mündlichen Sprachgebrauch geht.

Communicative Language Teaching: Diese Methode ist wohl die bekannteste und geläufigste Form der didaktischen Vermittlung im FSU. Kontextualisiertes, kommunikatives und kreatives Lernen steht hier im Zentrum. Ziel ist es, Sprache nicht mit Hilfe eines kognitiv-logischen Trainings zu vermitteln, sondern den Lerner auch auf einer emotionalen Ebene zu erreichen. Die LS sollte im Unterricht nicht präsent sein und Übersetzung als Übungsform ist nicht vorgesehen. In weniger puristischen Auslegungen dieser Methode wird Übersetzungen im Unterricht eine kleine Rolle zugestanden: „Translation may be used where students need or benefit from it." (Finocchiari and Brumfit, zitiert nach Richards \& Rodgers, 2001, p. 156). Eine systematische Einbindung von Übersetzungsübungen oder der LS in den Unterricht findet ebenso wenig statt, wie eine genauere Definition, was genau ein sinnvoller Gebrauch von Übersetzung sei und wann die Lernenden davon profitieren können.

\section{4 - Argumente für die Verwendung der LS und Übersetzen als Übungsform im FSU}

Dieser kurze Abriss hat Folgendes deutlich gemacht: Die Übersetzungs-GrammatikMethode wurde zu Recht kritisiert, auch wenn bei rückblickender Beurteilung berücksichtigt werden sollte, dass sich diese Lehrform in erster Linie auf den Erwerb von Latein und Altgriechisch bezog und schon allein deshalb keine kommunikative Ausrichtung haben konnte. Festzuhalten ist jedoch, dass die Methode tatsächlich einen 
ASCHER, Claudia - Übersetzen im Fremdsprachenunterricht.

Para lá da tarefa: implicar os estudantes na aprendizagem de línguas estrangeiras no ensino superior.

Porto: FLUP, 2019, pp. 25-43

DOI: https://doi.org/10.21747/9789898969217/paraa2

großen Teil sprachlicher Fähigkeiten und Kompetenzen ignorierte, die in den verschiedenen Methoden der Gegenbewegung dann besonders betont wurden. Die methodisch-didaktischen Reaktionen auf diese Methode fielen teilweise sehr radikal aus und hatten zur Folge, dass die Übersetzung, die unmittelbar mit der Methode verbunden war, als Vermittlungs- und Übungsform zunächst aus einer Oppositionshaltung heraus fast vollständig aus dem FSU verdrängt wurde. Nur zaghaft fand die Übersetzung und die LS lediglich in den moderaten Ansätzen im Communicative Language Teaching Eingang in den FSU.

Bereits seit der zweiten Hälfte des vergangenen Jahrhunderts gibt es wiederum eine Gegenreaktion auf die Verdrängung der LS aus dem FSU und auch am Beginn des 21. Jahrhunderts scheinen Überlegungen zur Verwendung und Aufwertung der LS und zur Übersetzung als Übungsform im FSU eine Renaissance zu erleben (Rodrigues 2000, Butzkamm \& Caldwell 2009, Cook 2010, Cook \& Hall 2012, Tsagari \& Floros 2013); Butzkamm \& Caldwell (2009) sprechen in diesem Zusammenhang von einem Paradigmenwechsel.

Tatsächlich gibt es gute Argumente, die dafür sprechen, der LS allgemein und Übersetzungen im Besonderen einen Platz im FSU einzuräumen, ohne dabei jedoch kommunikativ-handlungsorientierte Ziele und den dominierenden Gebrauch der FS aufzugeben. Wie bereits in der Einleitung erläutert, geht es mir nicht um eine Alternative zum kommunikativen Unterricht, sondern um Formen zu dessen sinnvoller Erweiterung.

Die Argumente für den Gebrauch der LS im FSU lassen sich dabei in unterteilen

1. lernpraktische Argumente

2. lernpsychologische Argumente

3. emotional-motivationale Argumente

\section{1 - Lernpraktische Argumente}

Das erste Argument für Übersetzungsübungen im FSU ist ein sehr praktisches: Durch Übersetzungsübungen erlernen die Studierenden gezielt und gelenkt den Umgang mit dem Wörterbuch. Überlässt man die Wörterbucharbeit - die ja in jedem Fall stattfindet, ob nun zu Hause oder im Unterricht, mit online-Produkten oder mit analogen Papierwörterbüchern - dem Zufall, treten häufig Schwierigkeiten auf, da der Umgang mit diesen Nachschlagewerken in der Schule in der Regel nicht genug geübt wurde. Sowohl zweisprachige, besonders aber einsprachige Wörterbücher bereiten den Studierenden oft Probleme. Die Schwierigkeiten sind vielfältig und reichen von mangelndem Training, die alphabetische Reihenfolge von Schlagwörtern schnell und sicher zu bestimmen und 
ASCHER, Claudia - Übersetzen im Fremdsprachenunterricht.

Para lá da tarefa: implicar os estudantes na aprendizagem de línguas estrangeiras no ensino superior.

Porto: FLUP, 2019, pp. 25-43

DOI: https://doi.org/10.21747/9789898969217/paraa2

dementsprechend das gesuchte Wort zu finden, gehen über die korrekte Interpretation der im Wörterbuch verwendeten Symbole und Zeichen und reichen bis hin zu der Annahme, dass die erste im zweisprachigen Wörterbuch angegebene Übersetzung im Normfall die richtige sei.

Bei der Benutzung des zweisprachigen Wörterbuchs fehlt es in erster Linie an Kenntnissen, welche Hilfestellungen ein solches Nachschlagewerk bieten kann und die Einsprachigkeit wird oft mit Nutzlosigkeit gleichgesetzt. Dass sich neben Synonymen und hilfreichen lexikalischen Erklärungen auch beispielsweise Konjugations- und Deklinationstabellen und Satzmuster in einem einsprachigen Wörterbuch finden lassen, ist den wenigsten bekannt. Durch eine systematisch eingeführte und regelmäßig geübte Wörterbucharbeit lässt sich nicht nur diese Fehleinschätzung beheben und dadurch die (vor allen Dingen schriftliche) Performance der Studierenden in der FS verbessern. Die Vorstellung, dass der erste Eintrag im Wörterbuch der richtige sei, lässt sich auch durch gezielte Lexik-Übungen leicht widerlegen und trägt damit dazu bei, der Idee einer kontextunabhängigen 1:1-Übersetzung entgegenzuwirken. Dadurch wird auch ebenjenes Argument entkräftet, das häufig gegen die Verwendung von Übersetzung als Übungsform verwendet wird: Der Vorwurf, das Übersetzen fördere und festige den Gedanken vieler Studierender, es gebe kontextunabhängige und eindeutige lexikalische Entsprechungen zwischen LS und FS.

Cook \& Hall (2012) verweisen auf zahlreiche Arbeiten, die von der Übersetzung als fünfte Fertigkeit („fifth skill“) in der FS-Kompetenz sprechen (Cook \& Hall, 2012, p. 283). Im universitären Kontext und in Hinblick auf die dort vielerorts vorhandenen Masterstudiengänge im Bereich Übersetzen erhält diese Einschätzung eine sehr konkrete Dimension: Da die Studierenden schon im Bachelor-Studiengang ein wenig in die Übersetzungspraxis „hineinschnuppern“ können, erhalten sie bereits einen kleinen Eindruck von dieser Tätigkeit und können so in ihrer Wahl eines eventuellen MasterStudiengangs unterstützt werden. Dabei kann sowohl das Interesse an einem MasterStudiengang im Bereich Übersetzung geweckt werden, als auch die Entscheidung reifen, dass dieses Berufsfeld keine Option für den oder die Studierende darstellt. Auch wenn die Darbietung dieser Übungsform weitestgehend in didaktisierter Form geschieht, hat meine Erfahrung gezeigt, dass sie trotzdem dazu geeignet ist, unschlüssige Studierende in ihrer Wahl zu ermutigen.

\section{2 - Lernpsychologische Argumente}


ASCHER, Claudia - Übersetzen im Fremdsprachenunterricht. Para lá da tarefa: implicar os estudantes na aprendizagem de línguas estrangeiras no ensino superior. Porto: FLUP, 2019, pp. 25-43 DOI: https://doi.org/10.21747/9789898969217/paraa2

Es gibt eine Reihe von Forschungsarbeiten, die belegen, das Übersetzen auch aus lernpsychologischer Sicht von Vorteil für Lernende einer FS sein kann. Cook \& Hall (2012) geben einen umfassenden Überblick über den Forschungsstand auf diesem Gebiet, insbesondere den Englisch-Unterricht betreffend. Sie verweisen unter anderem auf eine Studie von Cummings aus dem Jahr 2007 (Cook \& Hall, 2012, p. 288), die zu dem Ergebnis kam, dass Übersetzung im FSU zu einem verbesserten metalinguistischen Bewusstsein bei den Lernenden führe. Diese Bewusstmachung führt laut Cummings wiederum zu einem effektiveren Unterricht und zu einem Ausbau von Lernstrategien auf Seiten der Lernenden. Andere Studien bestätigen, dass die Merkfähigkeit gesteigert werde, wenn Übersetzung als Lernstrategie angewandt werde. Der „Arbeitsspeicher“ des Gehirns werde weniger belastet als bei einem rein einsprachigen Zugang, so dass kognitive Prozesse einfacher und schneller ablaufen könnten (Cook \& Hall, 2012, p. 290).

Dass die Verwendung der LS und gezielte Übersetzungsübungen auch effizient beim Vokabellernen und der Wortschatzerweiterung helfen, legen andere Untersuchungen nahe. So betrachtet Lutjeharms (2003) beispielsweise die LS (in ihrer Terminologie L1) in diesem Kontext als sinnvolle Lernhilfe und sieht, dass die „Angst vor einer Förderung der Interferenz [...] nicht begründet [ist], weil nach heutigen Erkenntnissen nach der L1 erworbene Sprachen stark mit der L1 im mentalen Lexikon verbunden sind und bei der Sprachverwendung eine automatische Aktivierung von Übersetzungsäquivalenten im mentalen Lexikon wahrscheinlich ist." (Lutjeharms, 2003, p. 134). Auch Cook \& Hall (2012) verweisen auf eine ganze Reihe von Arbeiten, die die Verwendung der LS als vorteilhaft für den Erwerb und das Training von neuem Vokabular bewerten (Cook \& Hall, 2012, p. 290).

\section{3 - Emotional-motivationale Argumente}

Die dritte Gruppe von Argumenten für den Gebrauch der LS und der Übersetzung im FSU rückt die emotionale Ebene beim Lernen einer FS und die Motivation der Studierenden in den Mittelpunkt. Welchen Beitrag können Übersetzungsübungen und die LS leisten, um die Motivation der Studierenden zu fördern?

Die Benutzung der LS von Seiten der Lehrkraft ist natürlich nicht immer möglich, zum Beispiel in sprachlich heterogenen Gruppen oder einfach, weil der Lehrende der LS nicht mächtig ist. Falls es jedoch durchführbar ist, bietet der Gebrauch der LS durch die Lehrkraft meiner Meinung nach einige Vorteile, die sich positiv auf die Motivation der Studierenden auswirken und diese aktiv in das Unterrichtsgeschehen einbinden können. 
ASCHER, Claudia - Übersetzen im Fremdsprachenunterricht. Para lá da tarefa: implicar os estudantes na aprendizagem de línguas estrangeiras no ensino superior. Porto: FLUP, 2019, pp. 25-43 DOI: https://doi.org/10.21747/9789898969217/paraa2

Der empathische Gebrauch und die bewusste Verwendung der LS von Seiten der Lehrkraft stellt eine Wertschätzung der Sprache und damit auch eines Teils der Identität der Lernenden dar. Diese Form der Anerkennung steigert in der Regel die Motivation der Studierenden und mindert das sprachliche Machtgefälle, welches im Klassenraum zwischen Lehrkraft und Studierenden existiert. Dies schafft Vertrauen auf Seiten der Lernenden und ermöglicht ein entspannteres und angstfreies Lernen. Gerade für Studierende aus Portugal hat dieser Punkt eine hohe Relevanz, weil sie erfahrungsgemäß aus einer recht autoritären und stark hierarchisierten Lernumgebung in der Schule kommen. Durch die gezielte Verwendung der LS kann ein Lernen „auf Augenhöhe" gefördert werden. Diese Abschwächung der klassenrauminternen Hierarchien kann zu einer gleichberechtigteren und motivierenderen Lernatmosphäre beitragen. Die Einbindung der LS gibt den Lernenden zudem die Möglichkeit, sich aktiv in den Unterricht einzubringen auch sprachlich ihren ganz persönlichen Erfahrungen zu schöpfen und sowohl über die eigenen als auch über die FS zu reflektieren. Ich selbst sehe meine Studierenden selten engagierter über Sprache diskutieren, als wenn sie gemeinsam eine Übersetzungsaufgabe lösen.

Dass „authentische Kommunikation“ im Unterricht ein Faktor ist, der sich positiv auf die Motivation der Lerner auswirkt, wird beispielsweise von Kara bestätigt (Kara, 2007, p. 63). Gleichzeitig präzisiert sie aber auch unter Berufung auf Kaikkonen (2002), was Authentizität in diesem Kontext genau bedeute: „Der Begriff der Authentizität wird oft mit Echtheit gleichgestellt. Authentizität findet sich aber nicht in automatisch sog. authentischen Texten, sondern darin, wie authentisch der Text für den Schüler im Lernkontext ist. Authentizität ist also eine Erfahrung und der Schüler ist Täter, Urheber und Subjekt seines Lernens" (Kara, 2007, p. 63). In diesem Sinne halten die Studierenden am Ende einer Übersetzungsübung ein authentisches Resultat in den Händen, nämlich einen übertragenen Text, den sie selbst erarbeitet haben. Diese Erfahrung kann durchaus als lebensnaher wahrgenommen werden als beispielsweise ein nachgespielter Dialog, dessen „setting“ zwar in eine reale Situation eingebettet wurde, an dessen Ende jedoch lediglich eine (mehr oder weniger gelungene) Imitation dieser Situation steht.

An der Gestaltung eines authentischen Lernkontextes sind meines Erachtens aber nicht nur die Lernenden, sondern auch die Lehrenden beteiligt. Hier bedeutet Authentizität auch, dass der Lehrende, der die LS beherrscht, sie auch (dosiert) benutzt und nicht künstlich komplett aus dem Unterrichtsraum verbannt. Damit wird auch ein oft unbekannter Aspekt der Lehrerpersönlichkeit für die Studierenden sichtbar, nämlich 
ASCHER, Claudia - Übersetzen im Fremdsprachenunterricht.

Para lá da tarefa: implicar os estudantes na aprendizagem de línguas estrangeiras no ensino superior.

Porto: FLUP, 2019, pp. 25-43

DOI: https://doi.org/10.21747/9789898969217/paraa2

dessen Kompetenzen in der LS, und es kann ein Rollenwechsel bei der Kompetenzverteilung stattfinden. Die „Allwissenheit“ der Lehrkraft in der FS wird hier häufig umgekehrt - die Studierenden sind die „Experten“ ihrer eigenen Sprache und werden dadurch auf sehr aktive und herausfordernde Weise in den Unterricht eingebunden.

Last but not least sei hier noch der Spaßfaktor, den die Benutzung der LS und die Einbindung von Übersetzungsübungen im Unterricht mit sich bringen kann, als unterstützendes Argument aufgeführt. Gerade in niedrigeren Niveaustufen sind scherzhafte Anmerkungen in der Regel nur in der LS möglich. Sie können zur Auflockerung des Unterrichts beitragen und Unterrichtsklima wesentlich entspannter und damit motivierender gestalten.

\section{5 - Ein Beispiel aus der Praxis - Übersetzen im FSU}

Die vorangegangenen Ausführungen haben gezeigt, dass die Integration der LS ein motivierender Input für die Studierenden sein kann. Übersetzungsübungen stellen eine spezifische Form dieser Einbindung dar.

Die Aufwertung der Übersetzung im FSU spiegelt sich zunehmend auch in Lehrwerken bereits wider. Im Lehrwerk $D a F$ kompakt ${ }^{3}$ beispielsweise findet sich am Ende einer jeden Lektion die Rubrik "Sprachen der Welt“. Sie dient dazu, das Sprachbewusstheit der Lerner zu stärken, indem sie aufgefordert werden, ein Phänomen im Deutschen mit der vorgegebenen Übersetzung ins Englische und mit der eigenen Sprache zu vergleichen. Dabei können unterschiedliche Phänomene als Ausgangspunkt für den Sprachvergleich dienen: Wortschatz, idiomatische Wendungen oder Grammatikstrukturen. Auch in der überarbeiteten Fassung DaF kompakt neu existiert die Kategorie „Über Sprache reflektieren“. Auch hier wird, meist in tabellarischer Form und in allen Niveaustufen (bei progressiver Komplexität) ein Sprachvergleich zwischen Deutsch, Englisch, teilweise Französisch und der LS angestrebt, wie die beiden folgenden Beispiele aus dem Niveau A1 und Niveau B1 illustrieren:

\footnotetext{
3 Dieses Lehrwerk - und seit zwei Jahren die überarbeitete Version "DaF kompakt neu" - wird in Coimbra im Deutschunterricht im ersten und zweiten Studienjahr verwendet. Im dritten Studienjahr wird hauptsächlich mit selbsterstellten Lehrmaterialen gearbeitet.
} 
ASCHER, Claudia - Übersetzen im Fremdsprachenunterricht.

Para lá da tarefa: implicar os estudantes na aprendizagem de línguas estrangeiras no ensino superior.

\section{2 Über Sprache reflektieren}

Ergänzen Sie die Tabellen. Wie heißen die Wörter in Ihrer Sprache? Vergleichen Sie im Kurs.

\begin{tabular}{|c|c|c|}
\hline Deutsch & Englisch & andere Sprache(n) \\
\hline kommen & to come & \\
\hline sprechen & to speak & \\
\hline lernen & to learn & \\
\hline studieren & to study & \\
\hline arbeiten & to work & \\
\hline sein & to be & \\
\hline
\end{tabular}

$\frac{\frac{\text { Deutsch }}{\mathrm{er}}}{\frac{\text { sie (Singular) }}{\text { sie (Plural) }} \frac{\text { he }}{\text { she }}}-\frac{\text { andisch Sprache(n) }}{\text { they }}-$

Abbildung 1. Auszug aus dem Lehrwerk DaF kompakt neu A1, Arbeitssteil, Seite 82.

\section{2 Über Sprache reflektieren}

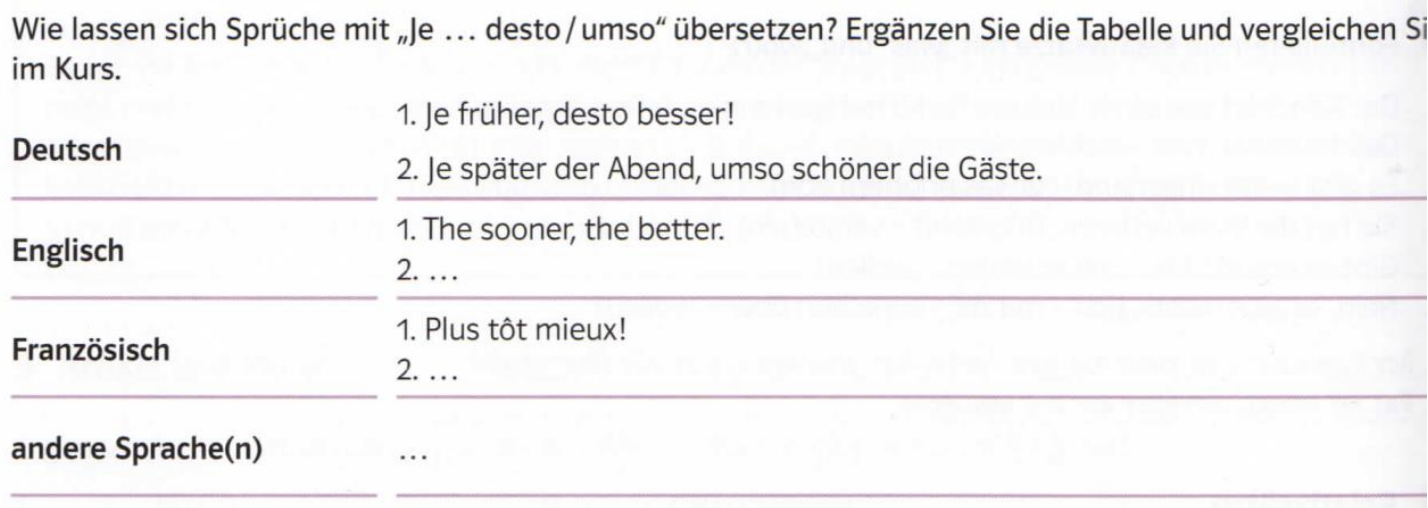

Abbildung 2. Auszug aus dem Lehrwerk DaF kompakt neu B1, Arbeitssteil, Seite 196.

An diese kleinen, das Sprachbewusstsein fördernden Einheiten sind die Studierenden also von Beginn ihrer Ausbildung an gewöhnt. Im dritten Jahr ihres Studiums wende ich dann eine der drei pro Woche zur Verfügung stehenden Unterrichtsstunden für eine didaktisierte Form der Übersetzung auf, die ich im Folgenden anhand eines Beispiels erläutern möchte.

Grundsätzlich verwende ich keine einzelnen Sätze, sondern Texte oder Textauszüge, um den zu übersetzenden Abschnitt zu kontextualisieren und den Ausgangstext auch beispielsweise als Sprechanlass oder für eine andere Form der Übersetzung, nämlich eine (mündliche) Zusammenfassung in der FS, nutzen zu können. In der Regel werden pro Unterrichtseinheit mehrere zusammenhängende Sätze übersetzt, die eventuell von mir zuvor bearbeitet wurden. Zunächst geschieht die Übersetzung im Plenum unter Anleitung der Lehrkraft, in einem weiteren Schritt autonom in Kleingruppenarbeit und, 
ASCHER, Claudia - Übersetzen im Fremdsprachenunterricht. Para lá da tarefa: implicar os estudantes na aprendizagem de línguas estrangeiras no ensino superior. Porto: FLUP, 2019, pp. 25-43 DOI: https://doi.org/10.21747/9789898969217/paraa2

wenn das Verfahren bereits ausreichend gemeinsam eingeübt wurde, auch in Einzelarbeit zu Hause.

Das Grundprinzip besteht darin, die jeweils zu übersetzende Einheit (in der Regel einen Satz) in einer vorgegebenen Sequenz zu übertragen, die in syntaktische, semantische, grammatische und stilistische Gesichtspunkte unterteilt ist. Um diese Aufteilung zu veranschaulichen, gebe ich den Studierenden ein vierstufiges Modell vor, welches sich am menschlichen Körper orientiert: Knochen, Muskeln, Kleidung und Styling. Um in einer fortgeschrittenen Phase auch schnell und wortlos kommunizieren zu können, wird allen vier Kategorien jeweils eine Geste zugeordnet. Die Knochen werden mit vor dem Gesicht gekreuzten Unterarmen dargestellt, die Muskeln symbolisiert ein angewinkelter Oberarm um den Bizeps zu betonen, bei der Kleidung zupft die Lehrkraft an einem Teil ihrer Oberbekleidung und das Styling wird mit einer frisierenden Geste am eigenen Haarschopf demonstriert. Diese Gesten können selbstverständlich abgewandelt werden, wichtig ist allein, dass dadurch eine schnelle nonverbale Kommunikation bei der Übersetzungsarbeit und eventuell auch zur (Auto)Korrektur ermöglicht wird. Außerdem ist diese aus der Mnemotechnik entlehnte die Verbindung von Geste und personifizierten Kategorien für die Studierenden hilfreich, sich die erforderlichen Schritte in der richtigen Reihenfolge einzuprägen.

Die Knochen symbolisieren dabei die grundlegende syntaktische Struktur eines Satzes. Wie ein Skelett den menschlichen Körper stützen diese Strukturen den kompletten Satz, sind fest und wenig flexibel. Zu diesen Stützen zähle ich die Bestimmung und Einteilung der Einheit in Haupt- und Nebensatz, damit verbunden eventuell vorhandene Kommata, die Verbposition inklusive Verbklammer und das Subjekt. Diese Elemente werden zunächst notiert.

Die Muskeln sind alle anderen Teile des Satzes: Ergänzungen, Angaben, Adjektive, Adverbien usw. - sie geben dem Satz das „Fleisch“, die Informationen, die der Satz transportiert, werden bestimmt. An diesem Punkt steht die grammatische Korrektheit noch nicht im Zentrum der Aufmerksamkeit, kann aber, wenn von den Studierenden angesprochen, thematisiert werden. Auch diese Elemente werden zunächst in eine mögliche Position im Satz geschrieben.

Beim Thema Kleidung steht nun die Grammatik im Mittelpunkt. Bei der Kleidung kann es verschiedene Fehlerquellen geben: eine nicht optimal Zusammenstellung der Farben oder die falsche Größe beispielsweise. Ähnlich verhält es sich mit der Anwendung grammatischer Regeln: Kasus, Genus, Deklinationen, Konjugationen usw. können nicht korrekt benutzt worden sein oder fehlen und können an dieser Stelle korrigiert werden. 
ASCHER, Claudia - Übersetzen im Fremdsprachenunterricht. Para lá da tarefa: implicar os estudantes na aprendizagem de línguas estrangeiras no ensino superior. Porto: FLUP, 2019, pp. 25-43 DOI: https://doi.org/10.21747/9789898969217/paraa2

Nach diesen drei Schritten sollte bereits eine syntaktisch, semantisch und grammatisch korrekte Übersetzung weitestgehend gewährleistet sein. Das abschließende „Styling“ dient nun dazu, stilistische Optionen abzuwägen, eventuell auch um noch semantische Alternativen einzelner Elemente zu diskutieren. Dieser Aufbau erleichtert das Übersetzen nicht nur deshalb, weil schrittweise vorgegangen wird, er schärft auch das Bewusstsein für die verschiedenen Stufen und die damit verbundenen Funktionen der einzelnen Elemente im Satz.

Im Folgenden will ich das Verfahren an einem Beispiel demonstrieren. Der Ausgangstext ist der von mir bearbeitete Beginn des portugiesischen Wikipedia-Artikels über das Internet. Nach einer Vereinfachung lautet der erste Satz wie folgt:

(1) A Internet é um sistema global de redes de computadores que utilizam protocolos.

Im ersten Schritt wird nun also das Skelett, die Knochen, freigelegt. Im Plenum wird darüber diskutiert, welche Satzarten im Beispiel enthalten sind, Subjekt und Verben werden identifiziert. Die Lehrkraft kann die Diskussion anregen und unterstützende Fragen stellen, die Analyse sollte aber in erster Linie bei den Studierenden liegen. Der Lehrende untermalt dabei seine Fragen und Hinweise mit oben beschriebener Geste. Dies gilt für den gesamten Prozess der Übersetzung. Im Ergebnis wird die Gruppe zu dem Schluss kommen, dass es sich um einen Haupt- und einen Nebensatz handelt (gegebenenfalls kann hier schon die Art des Nebensatzes bestimmt werden), dass a internet das Subjekt und é und utilizam die verwendeten Verben sind. Diese Elemente werden nun im Plenum übersetzt, deren Position im Satz bestimmt und an der Tafel (später dann im eigenen Heft) notiert. Grammatische Fehler, sollten sie auftreten, werden in diesem Schritt noch nicht korrigiert. Im hier gezeigten authentischen Beispiel wurde beispieslweise das Genus des Subjekts nicht korrekt identifiziert:

(2) Die Internet ist benutzen.

Sowohl das Komma als auch Subjekt und Verben befinden sich aber bereits an der passenden Position. Im zweiten Schritt, in meinem Modell die „Muskeln“, werden die fehlenden Elemente des Satzes ergänzt und an passender Stelle eingefügt. Nach Analyse des Hauptsatzes und Diskussion im Plenum, bei der bereits das Genus des Subjekts korrigiert wurde, kam die Gruppe zu folgendem Ergebnis: 
ASCHER, Claudia - Übersetzen im Fremdsprachenunterricht. Para lá da tarefa: implicar os estudantes na aprendizagem de línguas estrangeiras no ensino superior. Porto: FLUP, 2019, pp. 25-43 DOI: https://doi.org/10.21747/9789898969217/paraa2

(3) Das Internet ist ein globaler System von Netze von Computer, benutzen.

Beim Nebensatz stellten die Studierenden zunächst fest, dass es sich um einen Relativsatz handelt und ergänzten das Relativpronomen:

(4) Das Internet ist ein globaler System von Netze von Computer, die benutzen.

In der folgenden Diskussion wurde bereits das Genus von „System“ korrigiert und die damit verbundene Korrektur der Adjektivdeklination vorgenommen. Ebenfalls wurde erkannt, dass es sich bei „Von Netzen“ um Dativ Plural handelt und das „n“ am Ende des Substantivs dementsprechend ergänzt. Der „Muskelaufbau“ wurde schließlich wie folgt abgeschlossen:

(5) Das Internet ist ein globales System von Netzen von Computer, die protokolle benutzen.

Beim folgenden Abschnitt „Kleidung“ steht die grammatische und orthografische Korrektur im Vordergrund. Die vorangegangenen Beispiele haben aber auch gezeigt, dass sich diese Aktivität nicht auf diese Kategorie beschränkt, sondern von einigen Studierenden bereits im Vorfeld - zumindest teilweise - durchgeführt wird. Sollte dies der Fall sein, kann die Korrektur selbstverständlich sofort eingearbeitet werden. Die Korrekturen von (5) wurden schrittweise vorgenommen, wie Satz (6) und (7) zeigen:

(6) Das Internet ist ein globales System von Netzen von Computer, die Protokolle benutzen.

(7) Das Internet ist ein globales System von Netzen von Computern, die Protokolle benutzen.

Auch im letzten Abschnitt, dem „Styling“, erfolgte eine schrittweise Modulierung des Satzes. Zunächst wurde erfolgreich eine Alternative zu der Formulierung „von Netzwerken von Computern" gefunden und danach das Adjektiv „global“ durch "weltweit“ ersetzt:

(8) Das Internet ist ein globales System von Computernetzen, die Protokolle benutzen. 
ASCHER, Claudia - Übersetzen im Fremdsprachenunterricht. Para lá da tarefa: implicar os estudantes na aprendizagem de línguas estrangeiras no ensino superior. Porto: FLUP, 2019, pp. 25-43 DOI: https://doi.org/10.21747/9789898969217/paraa2

(9) Das Internet ist ein weltweites System von Computernetzen, die Protokolle benutzen.

Zum Vergleich der Ausgangssatz:

(1) A Internet é um sistema global de redes de computadores que utilizam protocolos.

Das Verfahren wurde in ähnlicher Form dann auf den weiteren Text angewandt. Dabei hat sich die Lehrkraft zunehmend aus der Plenumsdiskussion zurückgezogen und nur noch punktuell und mit den vereinbarten Gesten als Hilfestellung ins Geschehen eingegriffen. Bemerkenswert war hierbei auch die Tatsache, dass die Studierenden oft eigenständig eine Arbeitsteilung vornahmen und einzelne Phänomene (Genus, Orthographie, Wortbedeutung) in Kleingruppen prüften. Größere syntaktische und grammatische Zusammenhänge wurden weiterhin in der Gruppe diskutiert.

\section{6 - Fazit}

Meine anfängliche Skepsis dem Einsatz von Übersetzungsübungen im FSU ist einer überwiegend positiven Einschätzung gewichen. Im Zuge der Übersetzungseinheiten habe ich deshalb das vorgestellte didaktische Modell zur Übersetzung entwickelt, das jedoch noch einer soliden empirischen Prüfung unterzogen werden sollte.

Die Anwendung des von mir vorgeschlagenen stufenweisen und personifizierten Übersetzens hat in der Praxis gezeigt, dass Übersetzen im FSU nicht nur theoretisch positive Auswirkungen auf Performance und Motivation der Studierenden hat. Zu beachten ist jedoch, dass die Lehrkraft bei diesem Vorgehen häufig den zu bearbeitenden Text zuvor der speziellen Übersetzungsmethode anpassen muss, das heißt in der Regel, dass er in allen vier Kategorien vereinfacht und weniger komplex gestaltet werden muss.

Bereits nach wenigen Einheiten konnte ich feststellen, dass die Studierenden ihre Kompetenzen besonders im analytischen Bereich stark verbessern konnten. Sie waren mit dem vierstufigen Modell nicht nur in der Lage, gute Ergebnisse bei der Übersetzung selbst zu erzielen, sie konnten mit den erworbenen Strategien ebenfalls ihre eigene Textproduktion zunehmend selbständig einer kritischen Analyse unterziehen und gegebenenfalls korrigieren. Insbesondere bei Arbeiten in der Gruppe waren die Studierenden aktiv und motiviert am Unterrichtsgeschehen beteiligt, welches sie zunehmend selbst steuern und bestimmen konnten, da sie durch den vorgegebenen 
ASCHER, Claudia - Übersetzen im Fremdsprachenunterricht. Para lá da tarefa: implicar os estudantes na aprendizagem de línguas estrangeiras no ensino superior. Porto: FLUP, 2019, pp. 25-43 DOI: https://doi.org/10.21747/9789898969217/paraa2

Rahmen und die trainierten einzelnen Schritte schon nach kurzer Zeit selbständig in der Lage waren, die Ausgangstexte zu analysieren, zu übersetzen und grammatisch und stilistisch zu korrigieren und zu verfeinern. Dies wiederum trug zur Motivation und zum Engagement der Studierenden bei. Die eingeführten Gesten unterstützten und belebten zudem die Kommunikation und wurden nicht nur von der Lehrkraft, sondern auch von den Studierenden selbst verwendet. Durch die personifizierten Schritte konnten sie sich sowohl die einzelnen Stufen selbst als auch deren Abfolge gut einprägen. Zudem eignet sich diese Übungsform, um die Arbeit im Team zu stärken und zu fördern und damit nicht nur sprachliche Fähigkeiten, sondern auch den sozialen Zusammenhalt und die Zusammenarbeit in der Gruppe positiv zu beeinflussen. Die Aufwertung der LS durch die Verwendung von Texten in der LS und die damit verbundene Erfahrung der eigenen Kompetenz, was beispielsweise die Textanalyse angeht, spiegelte sich in angeregten und fruchtbaren Diskussionen im Unterricht wider. Damit ist die Übersetzung Im FSU weit mehr als ein Instrument zur intellektuell-kognitiven Schulung, als das sie lange zu Unrecht gesehen wurde und sollte einen angemessenen Platz im FSU bekommen.

\section{Bibliographie}

Butzkamm, W. \& J. Caldwell (2009). The bilingual reform: A paradigm shift in foreign language teaching. Tübingen: Narr Studienbücher.

Cook, G. (2010). Translation in Language Teaching: An Argument for Reassessment. Oxford: Oxford University Press.

Cook, G. \& Hall, G. (2012). Own-language use in language teaching and learning. Language Teaching. Volume 45, Issue 3. Cambridge: Cambridge University Press.

Doff, S. (2016). Vermittlungsmethoden: Historischer Überblick. Handbuch Fremdsprachenunterricht. 6. völlig überarbeitete und erweiterte Auflage. Tübingen: Francke. 320-324.

Gündoğdu, M. \& Büyüknisan, E. (2012). Übersetzung im DaF-Unterricht. Tagungsbeiträge zum IX. türkischen Germanistik-Symposium „Wissen-Kultur-Sprache und Europa“-Neue Konstruktionen und Neue Tendenzen. Eskişehir. 188-197.

$\mathrm{Haß}$, F. (2010). Methoden im Fremdsprachenunterricht. Handbuch Fremdsprachendidaktik. Seelze-Velber: Klett/Kallmeyer. 151-156.

House, J. (2001). Übersetzen und Deutschunterricht. Deutsch als Fremdsprache. Ein internationales Handbuch, 1, Berlin et al.: Mouton de Gruyter. 258-268. 
ASCHER, Claudia - Übersetzen im Fremdsprachenunterricht. Para lá da tarefa: implicar os estudantes na aprendizagem de línguas estrangeiras no ensino superior. Porto: FLUP, 2019, pp. 25-43 DOI: https://doi.org/10.21747/9789898969217/paraa2

Kaikkonen, P. (2002). Identitätsbildung als Zielvorstellung im interkulturellen Fremdsprachenunterricht. Quo vadis foreign language education. Reports from the Department of Teacher Education in Tampere University. 33-44.

Kara, H. (2007). Mündliches Portfolio im DaF-Unterricht-ein Lehrexperiment anhand von Portfolioarbeiten. Informationen Deutsch als Fremdsprache. Heft 34 (1). 62-75.

Lutjeharms, M. (2003). Die Rolle der Übersetzung in die Ausgangssprache für den Wortschatzerwerb in der Fremdsprache. Zeitschrift für Interkulturellen Fremdsprachenunterricht, 8 (2). 128-139.

Richards, J. C. \& Rodgers, T. S. (2001). Approaches and methods in language teaching. 2. ed. Cambridge: Cambridge University Press.

Rodrigues, C. (2000). Warum hast du das so und nicht anders übersetzt? Übersetzen als Übungsform: praktische Beispiele aus dem brasilianischen Deutschunterricht. Fremdsprache Deutsch. 23, 25-32.

Tsagari, D. \& Floros, G. (Hrsg.). (2013). Translation in language teaching and assessment. Cambridge: Cambridge Scholars Publishing. 